\title{
Can Network Theory be the Gold Standard for Mathematicians?
}

\section{Yilun Shang*}

Institute for Cyber Security, University of Texas at San Antonio, San Antonio, Texas 78249, USA

Over the last decade or so, the study of complex networks has attracted a surge of interest in varied branches of science and industry, from engineering to economics, from neurobiology to statistical physics. Network theory emerging as a blooming science has been recognized as a network-based paradigm that is taking science by storm. Much progress has been achieved, but even more questions are being posed. At the dawn of the millennium, S.H.Strogatz [1] enumerated some possible complications facing network theory: (1) structural complexity; (2) network evolution; (3) connection diversity; (4) dynamical complexity; (5) node diversity; and (6) meta-complication. These challenges have resisted complete solutions so far. A special issue on complex systems and networks composed by leading scientists in the field was honored by Science in 2009, which highlights important sweeping advances as well as up-to-date insightful perspectives [2]. Albeit young, network theory was preceded by an old and profound subject, graph theory, in mathematics that governs the discrete universe.

Graph theory has a long history and the story begins with Leonhard Euler more than two and a half centuries ago. Euler successfully solved the Konigsberg's seven bridges problem, which asked if one could arrange a walk in such a way that he would cross each bridge once and only once. Without using a picture representing the geography of these lands and bridges, he reformulated it in a way that vertices were connected by edges, constituting a "graph". This work is inarguably recognized as the very source of modern graph (network) theory. Following in the footsteps of forerunners, nowadays, when we look at various complex networks, from the World-Wide Web to social systems, we abstract them as graphs despite conspicuous differences in the nature of vertices and interactions between them.

Many ideas and techniques that are used in modeling and analyzing networks can be traced back to the seminar works of P.Erd"os and A. R'enyi in 1950 's. The classical Erd"os-R'enyi random graph model only assumes that each edge appears between two vertices independently with some fixed probability. This simple model serves as a guiding light in the mist. As an influential and legendary mathematician, Erd"os had more than 500 collaborators and published around 1500 academic papers in his lifetime. Mathematicians are familiar with the famous Erd"os number, which measures the collaborative distance between a person and Erd"os. In the coauthorship networks of scientists, Erd"os himself is1 assigned an Erd"os number zero. Any other author's Erd"os number is one plus the lowest Erd"os number of any coauthor. In the mathematical community, a low Erd"os number is considered prestigious. (As a young mathematician working on network theory, I have an Erd"os number 4).

Today as I write down the title, I wish deep down for it to come true someday. Physics has taken up the gold standard for mathematicians for decades. Mathematics is deeply entangled with physics and the line between them has been getting blurred. Many developments in modern mathematics, especially in geometry and topology, benefit much from string theory and quantum field theory. As a worthy successor of physics, network theory definitely has a great expectation. But the question is: what's the role mathematicians play along this journey? Our pioneers have set us a brilliant example. We ought to feel proud of it. Now that researchers from computer science, biology, and physics are plugging away [3], we mathematicians must rise to the occasion and cannot afford to miss out on the prolific journey.

\section{References}

1. Strogatz SH (2001) Exploring complex networks. Nature 410: 268-276.

2. Butts CT (2009) Revisiting the foundations of network analysis. Science 325 414-416.

3. Barab'asi A-L (2012) The network takeover. Nat Phys 8: 14-16.
*Corresponding author: Yilun Shang, Institute for Cyber Security, University of Texas at San Antonio, San Antonio, Texas 78249, USA, E-mail: shylmath@hotmail.com

Received June 28, 2012; Accepted June 30, 2012; Published July 04, 2012

Citation: Shang Y (2012) Can Network Theory be the Gold Standard for Math-ematicians? J Applied Computat Mathemat 1:e110. doi:10.4172/2168-9679.1000e110

Copyright: $\odot 2012$ Shang Y. This is an open-access article distributed under the terms of the Creative Commons Attribution License, which permits unrestricted use, distribution, and reproduction in any medium, provided the original author and source are credited. 